\title{
O DESASTRE NA PERSPECTIVA SOCIOLÓGICA E PSICOLÓGICA ${ }^{1}$
}

\author{
Eveline Favero ${ }^{2}$ \\ Universidade Estadual do Oeste do Paraná, Cascavel-PR, Brasil \\ Jorge Castellá Sarriera \\ Universidade Federal do Rio Grande do Sul, Porto Alegre-RS, Brasil \\ Melina Carvalho Trindade \\ Domus - Centro de Terapia de Casal e de Família, Porto Alegre-RS, Brasil
}

\begin{abstract}
RESUMO. O presente artigo tem por objetivo revisar e discutir conceitos de desastre na perspectiva de autores da Sociologia e da Psicologia, no sentido de contribuir com as discussões atuais sobre a abrangência do termo. Foi utilizada revisão de literatura incluindo-se os conceitos dos principais teóricos sobre o tema. No âmbito da Sociologia a palavra desastre abrange fenômenos delimitados no tempo e no espaço, capazes de causar danos físicos, perdas, rupturas sociais e mudanças no funcionamento da rotina diária. Características como o contexto social vulnerável em que a demanda exceda a capacidade de resposta são importantes para determinar um desastre. No âmbito da Psicologia, o termo, que faz menção a eventos súbitos e com potencial traumático delimitados no tempo e no espaço, refere-se a fenômenos coletivos que geram alto grau de estresse e provocam consequências/reações psicológicas nos envolvidos. Conclui-se que, embora os conceitos sejam importantes para classificar e delimitar fronteiras, torna-se fundamental compreender o desastre dentro do contexto social, político e econômico onde ele ocorre. Essas variáveis permitem avaliar a dimensão psicossocial das suas consequências, de modo que os desastres situam-se tanto como objeto de interesse científico quanto como problema de relevância social.
\end{abstract}

Palavras-chave: Psicologia Social; Sociologia; trauma.

\section{THE DISASTER ON THE PSYCHOLOGICAL AND SOCIOLOGICAL PERSPECTIVE}

\begin{abstract}
The article aims to review and discuss the concepts of disaster in the Sociology and Psychology author's perspective, in order to contribute to the current discussions on the definition of the term. For this, we used a literature review including the concepts of the leading theorists on the disaster. It appears that the word disaster in Sociology covers those events delimited in time and space, causing physical damage, loss, social disruption and changes in the functioning of the daily routine, so that the demand exceeds the capacity of response within a vulnerable context. On the Psychology's field the term allude to sudden events, with potential traumatic, delimited in time and space, being the disasters also a collective event, generating high levels of stress and with psychological consequences/reactions. We conclude that, although the concepts are important for classifying and defining boundaries it is essential to understanding a disaster within the social, political and economic context where it occurs. These are variables that allow evaluating the psychosocial dimension of its consequences, so that disasters are as much an object of scientific interest, and as a problem of social relevance.
\end{abstract}

Keywords: Social Psychology; Sociology, trauma.

\section{EL DESASTRE EN LA PERSPECTIVA SOCIOLÓGICA Y PSICOLÓGICA}

RESUMEN. Este trabajo tiene como objetivo revisar y discutir conceptos de desastre en la perspectiva de autores de la Sociología y Psicología, contribuyendo en las discusiones actuales sobre la abarcadura del término. Fue utilizada revisión de literatura, incluyendo los conceptos de los principales teóricos sobre el tema. En el ámbito de la Sociología, la palabra desastre abarca fenómenos delimitados en el tiempo y en el espacio, capaces de causar daños físicos, pérdidas, rupturas sociales y cambios en el funcionamiento de la rutina diaria. Características como el contexto social

1 Agradecimentos: Ao Conselho Nacional de Desenvolvimento Científico e Tecnológico (CNPq) e a Fundação de Amparo a Pesquisa do Rio Grande do Sul (FAPERGS) pelo auxílio financeiro concedido, através de bolsa de pesquisa, durante a elaboração desse trabalho

2 Endereço para correspondência: Rua Santos Dumont, 391, ap. 01, Região do Lago, CEP 85.812-300 - CascavelPR, Brasil. E-mail: evelinefavero@yahoo.com.br. 
vulnerable en el cual la demanda excede la capacidad de respuesta son importantes para determinar un desastre. En el campo de la Psicología el término alude a eventos súbitos, con un potencial traumático, delimitados en el tiempo y en el espacio, siendo los desastres fenómenos colectivos generadores de un alto grado de estrés y con consecuencias/reacciones psicológicas en los involucrados. Se concluye que, aunque los conceptos sean importantes para clasificar y delimitar fronteras, es esencial la comprensión del desastre dentro del contexto social, político y económico donde se produce. Estas son las variables que permiten evaluar la dimensión psicosocial de sus consecuencias, de modo que los desastres se ubiquen tanto como objeto de interés científico, como problema de relevancia social.

Palabras-clave: Psicología Social, Sociología, trauma.

O artigo tem por objetivo rever e discutir definições de desastre a partir de concepções de autores de perspectiva sociológica e psicológica e assim contribuir com as discussões relativas à abrangência do termo. A palavra desastre provém do termo latino dis astro, que traz a ideia de um infortúnio grande ou inesperado, calamidade e fracasso total (Oxford English Dictionary, 1987). De acordo com Green (1996), a definição é útil no dia a dia, mas não distingue um desastre de outras adversidades. Assim, torna-se necessário aprimorar seu desenvolvimento conceitual.

A importância de discutir o conceito de desastre não decorre da necessidade de se defender uma definição hegemônica para o termo, visto que seria quase impossível expressar em um conceito as diferentes características encontradas nos mais variados processos de desastre. Tal importância, segundo Quarantelli (1998), está no fato de que o conceito é um referente central para a área de estudo, tanto na Psicologia quanto na Sociologia ou em outras disciplinas que possam contribuir nessa discussão a partir das mais diferentes perspectivas.

No âmbito da Psicologia, o interesse pelos desastres, segundo Puy e Romero (1998), foi suscitado no contexto da Segunda Guerra Mundial, em que os diversos organismos oficiais do governo norte-americano financiavam estudos em Psicologia do Trauma, com o objetivo de extrapolar os resultados obtidos em contextos de desastres, aplicando-os às situações bélicas. Não obstante, embora tenham muito em comum com estressores como as guerras, os desastres diferem dessas últimas em dimensões temporais (McFarlane \& Norris, 2006).

Na última década, o desenvolvimento da Psicologia no campo dos desastres tem dado ênfase à resiliência psicológica e aos comportamentos adaptativos diante dos novos cenários do pós-desastre. Atualmente, o foco de interesse tem se deslocado para as intervenções e avaliações com base na comunidade, dentro de princípios consistentes com os modelos públicos de saúde mental (Dodge, 2006; Reyes, 2006c). Observa-se também um grande empenho no desenvolvimento tanto de teorias quanto de ferramentas de avaliação e intervenção em desastres como, por exemplo, a intervenção em crise (Dass-Brailsford, 2010). A ênfase está no fortalecimento da resiliência comunitária, em consonância com as características culturais de cada contexto, no atendimento das necessidades psicossociais e no cuidado com a saúde mental tanto dos profissionais quanto dos seus beneficiários (Ager, 2006; Dass-Brailsford, 2010; Dodge, 2006).

A Psicologia dos Desastres ou Psicologia nas/em emergências e desastres, como tem sido denominada na América Latina, é uma área ainda em desenvolvimento no Brasil e um campo ainda jovem, inclusive nos países desenvolvidos (Reyes, 2006a). Em vista dos inúmeros acontecimentos que têm marcado a história de nosso país na última década, como os deslizamentos no Rio de Janeiro, enchentes em Santa Catarina e o incêndio da Boate Kiss em Santa Maria, profissionais da Psicologia têm se mobilizado no sentido de oferecer apoio psicossocial às vítimas e aos familiares, porém não há formação específica na área e a Psicologia dos Desastres não está incluída como disciplina no currículo dos cursos de psicologia, o que resulta em profissionais com pouca ou nenhuma qualificação para atuar em situações que envolvam adversidades extremas.

Recentemente (08/05/2013) o Conselho Federal de Psicologia publicou nota técnica sobre a atuação de psicólogos em situações de emergência e desastre relacionadas com a Política Nacional de Defesa Civil. Além das considerações referentes à regulamentação da profissão e aos aspectos éticos da prática profissional, a nota destaca que as situações de 
emergências e desastres têm implicado a mobilização de serviços públicos e iniciativas privadas e/ou complementares. Desse modo, faz-se necessário que os serviços, em seus territórios de abrangência, estejam preparados e organizados para participar ativamente de ações de prevenção, preparação, resposta e reconstrução nessa situações (CFP, 2013).

A Psicologia dos Desastres, segundo Reyes (2006b), é um campo da Psicologia que em situações de desastre atua de modo a oferecer respostas de curto prazo ao estresse agudo, enfatizando intervenções com base na comunidade. Enquanto área aplicada da Psicologia, utiliza técnicas como as da intervenção em crise, visando o gerenciamento do estresse e com ênfase na diminuição da excitação emocional, na resolução de problemas e na adoção de estratégias de coping efetivas. Ao mesmo tempo em que se aproxima da Psicologia do Trauma, a Psicologia dos Desastres dela também se distingue, de diferentes modos. As duas não são áreas intercambiáveis entre si, no entanto a Psicologia do Trauma tem muito a oferecer aos sobreviventes dos desastres, orientando e desenvolvendo ações com vista a reduzir a exposição à traumatização nesses contextos, ações que, na maioria das vezes, dizem respeito ao gerenciamento da situação de crise instalada com o desastre.

Quando se instala uma crise, inúmeras demandas podem surgir, como aquelas ligadas à sobrevivência humana, à infraestrutura, à moradia, à comunicação e outras. Para Reyes (2006a), os desastres costumam ocasionar enormes perdas materiais e humanas, as quais, na maioria das vezes, tendem a ser rapidamente esquecidas. Nesse universo de perdas, as pessoas afetadas podem ter perdido familiares, amigos, estrutura de apoio comunitário, trabalho e outros bens de valor para a sobrevivência. $O$ psicólogo dos desastres necessita ser um expert não apenas em trauma, mas também em perdas e em mudança comunitária. Seu trabalho envolve conhecer como trabalhar em e com comunidades de maneira coordenada e em colaboração com diferentes profissionais e organizações.

Apesar dos avanços e da diversidade dos aspectos investigados relativos aos desastres, as consequências traumáticas deste tipo de evento ainda são muito enfatizadas por teóricos da Psicologia, tanto em pesquisas quanto em buscar conceituar o termo (Davidson \& McFarlane, 2006; McFarlane \& Norris, 2006; Páez, Fernández \& Martín Beristain, 2001). Trazendo como exemplo, autores como McFarlane e Norris (2006) conceituaram desastre como "um evento com potencial traumático o qual é experimentado coletivamente, com início agudo e delimitado no tempo" (p. 04). Os autores referiram também que os desastres podem ser cíclicos e com interações, não podendo ser simplesmente representados por fases claramente definidas, e que o uso do termo "agudo" é relativo, uma vez que o ciclo de um desastre pode ter sim um período agudo, mas ter seu curso estendido ao longo do tempo. Assim se observa que, embora definam desastres como eventos agudos, esses autores reconhecem também que nem todos se desenvolvem da mesma maneira.

A definição de McFarlane e Norris (2006) é útil quando se trata de avaliar desastres de início súbito e com grande potencial de destruição, como é o caso dos terremotos, mas não pode ser aplicada a outros tipos de eventos com início lento e pouco delimitados no tempo, como ocorre com as secas, e que causam efeitos devastadores no estilo de vida e bem-estar das comunidades (Boeckner, Bosch \& Johnston, 2003; Boyd, Quevillon \& Engdahl, 2010; Logan \& Ranzijn, 2008; Staniford, Dollard \& Guerin, 2009). Embora McFarlane e Norris (2006) tenham enfatizado que um desastre não pode ser entendido como sinônimo de trauma, ainda assim consideram como uma das características deste tipo de evento o potencial para o trauma.

Cabe considerar que, além do potencial traumático, a exposição prolongada à ameaça de um desastre e a não previsibilidade do seu término - como ocorre com as secas - são situações geradoras de altos níveis de estresse, interferindo na saúde psicológica e influenciando a percepção de segurança quanto ao futuro (McFarlane \& Norris, 2006). As secas são desastres que se manifestam por mudança ambiental e rápido declínio econômico, com consequências prolongadas no tempo (Boyd et al., 2010). Elas também desencadeiam a perda de recursos de sobrevivência, como água e alimentos, o que, segundo Ünal-Karagüven (2009), expõe indivíduos e grupos a importantes níveis de estresse e, consequentemente, interfere em sua saúde e bem-estar.

É importante considerar que a perspectiva a partir da qual se analisa um evento modifica 
também a compreensão do risco de desastre a que uma determinada população está exposta, de modo a naturalizá-lo como um agente externo de caráter abrupto, por exemplo, ou compreendê-lo como parte de um contexto e expressão da vulnerabilidade social (Britton, 1986). Desse modo, não são apenas as características físicas de um evento que estão relacionadas com as suas consequências, mas principalmente o contexto em que ele ocorre. Neste caso, muda-se a perspectiva de olhar para o desastre, o qual não é compreendido apenas como um evento de início agudo, como propuseram McFarlane e Norris (2006), mas muito mais como uma expressão aguda da vulnerabilidade social.

\section{DESASTRES, EMERGÊNCIAS E ACIDENTES}

Quando se trata de definir desastre, muitas vezes surge a dúvida sobre quais fenômenos podem ser abrangidos pela definição e no que eles diferem de emergências e acidentes, por exemplo. Quarantelli (1998) enfatizou que não existe consenso sobre o conceito de desastre, o que poderia estar prejudicando os avanços no debate científico e, consequentemente, na definição de uma base conceitual mínima para a compreensão dos fenômenos como um todo.

Com o objetivo de delimitar conceitos relacionados, Britton (1986) considerou desastres, emergências e acidentes como períodos de crise social, caracterizados por diferentes graus de estresse coletivo. GarciaRenedo (2008) sistematizou as ideias de Britton e acrescentou o termo "catástrofe" como sendo o evento de maior grau de estresse coletivo.

De acordo com Garcia-Renedo (2008), acidente estaria no extremo de menor estresse coletivo, sendo um evento em que a ruptura aconteceria para um grupo muito específico de vítimas, enquanto numa emergência uma situação de crise interferiria nas atividades realizadas por um determinado grupo de pessoas. Por outro lado, um desastre implicaria num maior número de pessoas afetadas, assim como na ruptura da maioria das estruturas sociais e da infraestrutura comunitária, como acontece no caso dos terremotos. A autora acrescentou o termo catástrofes para se referir à ruptura de todas as estruturas sociais de uma sociedade, como, por exemplo, o que ocorreu no
Tsunami do Sudeste Asiático e no contexto das bombas atômicas de Hiroshima e Nagasaki.

Desse modo, a diferenciação entre cada um desses eventos se daria em torno de três critérios: número de pessoas implicadas, capacidade de resposta do sistema, ruptura e danos nos sistemas sociais (Garcia-Renedo, 2008). Tal diferenciação faz-se importante especialmente do ponto de vista operacional, em que declarar um evento como um desastre pode implicar em maior ou menor mobilização de recursos tanto para a própria gestão da crise quanto para as vítimas, que necessitam se organizar para enfrentá-lo. Por outro lado, um desastre não pode ser mensurado apenas pelo número de envolvidos e pela ruptura das estruturas sociais, pois esse tipo de evento não tem apenas atributos objetivos e passíveis de mensuração, mas também repercussões subjetivas, econômicas e sociais, as quais geralmente se estendem por um longo período de tempo.

Entre os atributos subjetivos estão o potencial de afetar as crenças das pessoas, ocasionando a perda do sentido de invulnerabilidade e da crença num mundo justo, e o de gerar ansiedade diante da possibilidade de morte e sentimento de insegurança quanto ao futuro (Garcia-Renedo, 2008; Paez, Fernández \& Martín Beristain, 2001; Reyes, 2006c).

Os desastres estão também relacionados a um contexto social vulnerável (Britton, 1986). Seus impactos são mediados pelos elementos que compõem a realidade onde eles ocorrem, abrangendo fatores de natureza econômica e política que interferem não apenas na capacidade de enfrentamento de suas consequências, mas na perpetuação dos múltiplos fatores que compõem uma situação de risco; ou seja, os desastres podem ser entendidos como um sistema com características dinâmicas, constituído por uma combinação de fatores de risco. Tais fatores desencadeiam processos crônicos, fundados sobre uma relação prévia entre elementos do ambiente e os sujeitos de risco (Bonzo et al., 2001).

\section{DEFINIÇÕES DE DESASTRE NA PERSPECTIVA DA SOCIOLOGIA E DA PSICOLOGIA}

Para rever e discutir diferentes definições de desastre foram buscados na literatura os 
principais conceitos utilizados por teóricos da Psicologia e da Sociologia que têm sido referenciados nos estudos da área. Embora se pretenda focalizar a concepção de desastre do ponto de vista psicológico, foram revisados conceitos da Sociologia, pois originalmente a pesquisa sobre desastres é do domínio dessa disciplina. Isso, consequentemente, influenciou outras áreas, como a Psicologia, uma vez que o campo dos desastres é reconhecidamente multidisciplinar (Kreps, 1984).

Neste trabalho foram reunidos os conceitos dos teóricos mais influentes, apesar de se reconhecer que existem inúmeras definições para o termo. Garcia-Renedo (2008), em seu trabalho de tese, fez uma revisão sistemática sobre os diferentes conceitos de desastre, ressaltando que somente na década de 1980 existiram mais de 40 definições. Neste artigo não foram consideradas para análise as definições operacionais, como as que são adotadas por organizações que atuam em desastres (Cruz Vermelha, Defesa Civil, por exemplo), pois elas partem, na maioria das vezes, de critérios aplicados de acordo com os interesses de trabalho de cada organização (Marchezini,
2009). A busca pelas definições foi feita considerando-se todo o período anterior ao ano 2013 e realizada tanto na base de dados Scielo quanto em teses e livros. Um trabalho remetia a outro, e assim sucessivamente. Buscavam-se trabalhos que discutissem conceitualmente a palavra desastre, excluíam-se aqueles que trouxessem definições operacionais, bem como aqueles que adotassem conceitos de outros autores. Neste sentido, optou-se por apenas trabalhar com o conceito a partir do próprio autor, mesmo que outros teóricos tenham adotado a mesma definição em publicações posteriores. Quando se tratava de um novo conceito ou de um trabalho que agregasse algum aspecto novo na definição de desastre, o trabalho era incluído nesse estudo. Constatou-se que na maioria dos casos os autores atuais têm adotado definições conceituais que remontam a anos atrás, sendo que as décadas de 1960, 1980 e 2000 têm se destacado na produção dos avanços conceituais.

$\mathrm{Na}$ tabela 01 estão reunidas as definições de desastre selecionadas como representativas dos principais desenvolvimentos teóricos sobre 0 tema no âmbito da Sociologia.

Tabela 1 - Definições de Desastre na Perspectiva Sociológica

\begin{tabular}{|c|c|c|}
\hline Autor (es), ano & Nível de Análise & Definição \\
\hline Fritz (1961, p. 312) & Social & $\begin{array}{l}\text { Eventos, observáveis no tempo e espaço, no qual as sociedades } \\
\text { ou suas subunidades (comunidades ou regiões) sofrem danos } \\
\text { físicos e perdas elou rupturas no funcionamento de sua rotina. } \\
\text { Ambas, causas e consequências desses eventos estão } \\
\text { relacionadas com as estruturas sociais e processos das } \\
\text { sociedades ou suas subunidades. }^{*}\end{array}$ \\
\hline Britton (1986, p. 254) & Social & $\begin{array}{l}\text { Um produto social como expressão da vulnerabilidade da } \\
\text { sociedade humana que depende da interação entre os seres } \\
\text { humanos e sua utilização do espaço físico e social. }{ }^{*}\end{array}$ \\
\hline
\end{tabular}

Nota. *Tradução nossa.

Observa-se que as definições propostas pela Sociologia são abrangentes no sentido de que diferentes tipos de eventos podem ser considerados desastres, não necessariamente aqueles de início súbito. Tais definições partem da avaliação de danos físicos e perdas, rupturas sociais e mudanças no funcionamento da rotina diária (Fritz, 1961). Nenhuma dessas definições faz menção explícita às implicações psicológicas dos desastres.

Ao conceituar desastres, Fritz (1961) fez referência à sua temporalidade, ou seja, considerou-os como eventos observáveis no tempo e no espaço. Em relação a este aspecto, Coêlho (2007) mencionou que, embora fenômenos como as secas, a fome, acidentes nucleares e químicos, conflitos sociais e epidemias tenham consequências desastrosas no longo prazo, não poderiam ser considerados desastres de acordo com essa definição, pelo fato de não serem limitados no tempo e no espaço. Nesse sentido, é importante ampliar a visão de desastre para além daqueles fenômenos ditos "naturais" e com características físicas evidentes, pois desastres são acontecimentos com implicações também do ponto de vista psicossocial.

Para Britton (1986), os desastres são um produto social, expressão da vulnerabilidade humana; ou seja, um desastre não seria um 
evento isolado que incide sobre a realidade, mas fruto da interação entre os seres humanos e a utilização do ambiente. O conceito de Britton é importante na medida em que destaca o fato de que os desastres não acontecem desvinculados do contexto social, ou seja, num contexto onde não há interação entre a pessoa e o ambiente não pode existir um desastre. Do mesmo modo, num contexto onde foram desenvolvidos mecanismos eficientes para o enfrentamento do risco, certamente um evento não terá as mesmas proporções que num contexto de maior vulnerabilidade.

No tocante à Psicologia, foram selecionadas três definições, conforme a tabela 02 .

Tabela 2 - Definições de Desastre na Perspectiva Psicossocial

\begin{tabular}{lcl}
\hline Autor (es), ano & Nível de Análise & \multicolumn{1}{c}{ Definição } \\
\hline Quarantelli (1985, p. 50) & Psicossocial & $\begin{array}{l}\text { Quando, numa ocasião de crise, a demanda por ação excede a } \\
\text { capacidade de resposta. A ênfase está no esforço coletivo para dar } \\
\text { conta da crise particular por meio da restauração das capacidades ao } \\
\text { nível das demandas. }\end{array}$ \\
McFarlane e Norris (2006, p.04) & Psicossocial & $\begin{array}{l}\text { Um evento com potencial traumático o qual é experimentado } \\
\text { coletivamente, com início agudo e delimitado no tempo. Desastres } \\
\text { podem ser atribuídos a causas naturais, tecnológicas e humanas. }\end{array}$ \\
García-Renedo, Gil Beltrán e & Psicossocial & $\begin{array}{l}\text { Uma situação traumática que gera um alto grau de estresse aos } \\
\text { indivíduos de uma sociedade ou uma parte dela, devido à ação de um } \\
\text { aglente em uma comunidade vulnerável (natural, humano ou uma } \\
\text { combinação de ambos), produzindo uma alteração no funcionamento, } \\
\text { tanto em nível comunitário como individual, assim como uma série de } \\
\text { reações e consequências psicológicas nas pessoas envolvidas. As } \\
\text { demandas criadas excedem os recursos habituais de respostas } \\
\text { disponíveis na comunidade. }\end{array}$ \\
\hline
\end{tabular}

Nota. *Tradução nossa.

No conceito de Quarantelli (1985), os termos demandas e capacidades podem ser aplicados às diferentes dimensões de um contexto de desastre (física, econômica, estrutural, social e de outras naturezas), sendo muito comumente aplicados no âmbito psicossocial. Sobre esse conceito de desastre, McFarlane e Norris (2006) argumentam que a definição de Quarantelli é, de certo modo, abrangente e flexível, ao mesmo tempo em que é abstrata para delimitar fronteiras. Os conceitos foram aqui classificados no nível de análise psicossocial, de modo a separá-los didaticamente. Quarantelli foi um sociólogo, no entanto a sua conceituação de desastre não se restringe a esse campo de saber.

A definição de McFarlane e Norris (2006) faz menção aos desastres como eventos delimitados no tempo, que são experimentados coletivamente. Em relação aos conceitos da Sociologia, a definição de MacFarlane e Norris evidencia duas características, ou seja, o início abrupto e o potencial traumático. No conceito estão também presentes diferentes causas para os desastres, como causas humanas, naturais e tecnológicas, o que torna a definição mais abrangente do ponto de vista da origem do evento; no entanto, desastres que não têm início agudo, como as secas, ou que carecem de potencial traumático (Coêlho, Adair \& Mocellin, 2004; Favero \& Diesel, 2008), não estariam sendo abrangidos por essa definição. A adoção do termo traumático por diferentes teóricos da Psicologia dos Desastres revela que, embora essa seja uma disciplina independente da Psicologia do Trauma, ainda carrega a sua influência na avaliação psicológica desse tipo de fenômeno, especialmente em países como os Estados Unidos, com teóricos como McFarlane e Norris (2006), e a Espanha, com Garcia-Renedo et al. (2007), para citar alguns exemplos.

A definição proposta por García-Renedo et al. (2007) volta a tratar do caráter traumático (neste caso um desastre "é" uma situação traumática). Os autores acrescentam o fato de a situação ser geradora de alto grau de estresse, tomando como base as ideias de Britton (1986) ao se referir às diferenças entre desastres, emergências e acidentes. Os autores apontam a vulnerabilidade e a alteração no funcionamento social como anteriormente descritas no âmbito da Sociologia (Britton, 1986), e acrescentam na sua definição as consequências e reações psicológicas sofridas pelos envolvidos. Fazem 
menção ao conceito proposto por Quarantelli (1985) quando este se refere a demandas versus capacidade de resposta comunitária.

Embora seja importante discutir do ponto de vista teórico a definição de desastre, Kreps (1984) afirma que o termo é apenas útil como um conceito síntese como tantos outros em ciências, chamando a atenção para o número de características relacionadas ao fenômeno e dando a estas uma "etiqueta". Não obstante, para o mesmo autor, são as subsequentes identificações e medidas dessas características que fazem o conceito teoricamente importante. Nessa direção, McFarlane e Norris (2006) fazem referência à importância dos atributos de um desastre, tais como o tipo de impacto (circunscrito ou difuso), a rapidez de início (aqui os autores consideram como importante o início súbito), a medida de advertência e a extensão da ameaça, além da gravidade do impacto (proporção da população afetada, natureza e magnitude dos estressores individuais $e$ coletivos).

Por outro lado, ainda é necessário ampliar as discussões sobre o termo para além de suas características físicas, uma vez que um desastre não pode ser entendido como um fenômeno estranho ao ambiente social. Ao conceituar desastre, faz-se necessário não apenas caracterizar o problema a que está se referindo, mas também possibilitar a compreensão e a reflexão deste em consonância com cada realidade específica. Os desastres não são apenas objeto de interesse científico, mas - e sobretudo - fenômenos de interesse social, de modo que se torna de fundamental importância analisar os diferentes fatores que possam estar contribuindo para a manutenção da vulnerabilidade e perpetuação dos processos de desastre.

\section{CONSIDERAÇÕES FINAIS}

A partir da revisão conceitual, constatou-se que desastre não é uma palavra simples de ser definida, uma vez que abrange diferentes eventos e/ou processos com características distintas. Além disso, um desastre não pode ser compreendido desvinculado do contexto no qual ele ocorre, mas devem ser consideradas as variáveis físicas, sociais, políticas, econômicas e outras que possam estar implicadas. Desse modo, faz-se uma tentativa de integrar as definições de diferentes teóricos, de maneira a ampliar a compreensão e suscitar reflexões sobre 0 termo. As diferentes definições aparecem interligadas, revelando haver uma clara aproximação entre a perspectiva sociológica e psicológica na compreensão desse fenômeno.

Para integrar as definições dos diferentes autores (da Sociologia e da Psicologia) considerando-se as especificidades de cada uma delas, propõe-se a definir o termo desastre como um processo que tem sua origem na interação entre seres humanos e seu contexto social (Britton, 1986), salientando-se que, mais do que um evento agudo, um desastre é a expressão aguda da vulnerabilidade em suas diferentes dimensões (física, social, ambiental, etc.). Os desastres desafiam a capacidade humana de resposta (Quarantelli, 1985), podendo trazer consigo perdas (Fritz, 1961) repentinas e prolongadas no tempo. Eles podem ser cíclicos ou escalonados, de início súbito e com grande potencial traumático (GarciaRenedo et al., 2007; McFarlane \& Norris, 2006); ou ainda, podem se desenvolver de maneira lenta, de modo a expor indivíduos e grupos a prolongados períodos de estresse (Favero, Sarriera, Trindade \& Galli, 2013). Apesar das diferenças quanto às suas características físicas, não são apenas essas diferenças, mas também o contexto no qual ocorrem os desastres, e, na maioria das vezes, o que determinará as suas consequências e a capacidade de um determinado grupo social para enfrentá-los são as circunstâncias que envolvem os processos do desastre. $O$ termo desastre já foi muito discutido por teóricos da Sociologia, trazendo importantes contribuições para o campo ao longo dos anos, mas ainda carece de discussões na área da Psicologia. A reflexão sobre o que essa ciência adota como definição de desastre faz-se especialmente importante no início do desenvolvimento dos estudos na área no Brasil, de modo a se refletir na abrangência das pesquisas e no desenvolvimento teórico e conceitual.

Por fim, os desastres são processos que, além de evidenciar a capacidade de enfrentamento de indivíduos e grupos, despertam para a necessidade de transformação da realidade social. Embora do ponto de vista científico os conceitos sejam necessários para classificar e delimitar fronteiras, reitera-se aqui como fundamental compreender um desastre 
dentro do contexto social, político e econômico no qual ele ocorre. São essas variáveis que permitem avaliar a dimensão das consequências desses processos na vida das pessoas, e é por esse motivo que os desastres são um tema de relevância social e objeto de interesse de diferentes disciplinas.

\section{REFERÊNCIAS}

Ager, A. (2006). Toward a consensus protocol for psychosocial response in complex emergencies. In G. Reyes, \& G. A. Jacobs (Eds.), Handbook of international disaster psychology: fundamentals and Overview (pp. 35-49). Westport, CT: Praeger.

Boeckner, L., Bosch, K. \& Johnston, C. E. (2003). Coping in stressful times during drought (Historical Materials, G1525). Recuperado em 01 de setembro, de 2013, de http://digitalcommons.unl.edu/extensionhist/1735

Bonzo, C., Castro, B., De Lellis, M., Samaniego, C. \& Tissera, E. (2001). Aportes psicosociales al concepto de riesgo. In E. Saforcada, \& Colaboradores, El "Factor Humano" en la salud pública: una mirada psicológica dirigida hacia la salud colectiva (pp. 130-141). Buenos Aires: PROA XXI.

Boyd, B., Quevillon, R. P. \& Engdahl, R. M. (2010). Working with rural and diverse communities after disasters. In $P$. Dass-Brailsford (Ed.), Crisis and Disaster counseling: lessons learned from hurricane Katrina and other disasters (pp. 149-163). Los Angeles: Sage.

Britton, N. R. (1986). Developing an understanding of disaster. Journal of Sociology, 22(2), 254-271.

Coêlho, A. (2007). Percepção de risco no contexto da seca: um estudo exploratório. Psicologia para a América Latina, 10. Recuperado em 01 de setembro, de 2013, de http://pepsic.bvsalud.org/scielo.php?script=sci_arttext\&pid= S1870-350X2007000200012\&lng=pt\&nrm=iso

Coêlho, A. E. L., Adair, J. G. \& Mocellin, J. S. P. (2004). Psychological responses to drought in Northeastern Brasil. Interamerican Journal of Psychology, 38(1), 95-103.

Conselho Federal de Psicologia/CFP (2013). Atuação de psicólogos em situações de emergências e desastres, relacionadas com a política nacional de Defesa Civil: Nota Técnica de 08 de maio de 2013. Brasília: Autor. Recuperado em 01 de setembro, de 2013, de http://site.cfp.org.br/documentos/nota-tecnica-sobreatuacao-de-psicologaos-em-situacoes-de-emergencias-edesastres-relacionadas-com-a-politica-de-defesa-civil/

Dass-Brailsford, P. (2010). Effective disaster and crisis interventions. In P. Dass-Brailsford (Ed.), Crisis and disaster counseling: lessons learned from hurricane Katrina and other disasters (pp. 213-228). Los Angeles: Sage.

Davidson, J. R. T. \& McFarlane, A. C. (2006). The extent and impact of mental health problems after disaster. Journal of Clinical Psychiatry, 67(2), 9-14.

Dodge, G. R. (2006). Assessing the psychosocial needs of communities affected by disaster. In G. Reyes \& Jacobs, G., Handbook of international disaster psychology: fundamentals and overview (pp. 65-91). Westport, CT/London: Praeger.

Favero, E. \& Diesel, V. (2008). A seca enquanto um hazard e um desastre: uma revisão teórica. Aletheia, 27, 198-209.

Favero, E., Sarriera, J. C., Trindade, M. C. \& Galli, F. (2013). In J. F. Leite \& M. Dimenstein (Orgs.), Psicologia \& Contextos Rurais (pp. 303-332), Natal: EDUFRN.

Fritz, C. E. (1961). Disaster. In R. K. Merton \& R. A. Nisbet (Eds.), Contemporary Social Problems (pp. 651-694). New York: Harcourt, Brace and World.

Garcia-Renedo, M. (2008). El 11-M. Un estudio sobre su impacto psicológico desde ele entorno familiar y escolar en alumnos de infantil y primaria. Tese de Doutorado, Universitat Jaume I, Castelló de la Plana, Castellón, Espanha. Recuperado em 01 de setembro, de 2013, de http://repositori.uji.es/xmlui/handle/10803/10526

Garcia-Renedo, M., Gil Beltrán, J. M. \& Valero Valero, M. (2007). Psicología y desastres: aspectos psicosociales. Castelló de la Plana: Publicacions de la Universitat Jaume I.

Green, B. (1996). Cross-national and ethnocultural issues in disaster research. In A. Marsella, M. Friedman, E. Gerrity, \& R. Surfield (Eds.), Ethnocultural aspects of posttraumatic stress disorder: issues, research, and clinical applications (pp. 341-361). Washington, DC: American Psychological Association.

Kreps, G. A. (1984). Sociological inquiry and disaster research. Annual Review of Sociology, 10, 309-330.

Logan, C. \& Ranzijn, R. (2008). The bush is dying: a qualitative study of South Australian farm women living in the midst of prolonged drought. Journal of Rural Community Psychology, 12(2). Recuperado em 01 de setembro, de 2013, http://wnw.marshall.edu/jrcp/VE12\%20N2/jrcp\%2012\%20 2\%20Logan\%20and\%20Ranzijn.pdf

Marchezini, V. (2009). Dos desastres da natureza a natureza dos desastres. In N. Valencio, M. Siena, V. Marchezini, \& J. Costa (Orgs.), Sociologia dos desastres: construção, interfaces e perspectivas no Brasil (pp. 48-57). São Carlos: Rima.

McFarlane, A. C. \& Norris, F. H. (2006). Definitions and concepts in disaster research. In F. H. Norris, S. Galea, M. J. Friedman, \& P. J. Watson (Eds.), Methods for disaster mental health research (pp. 3-19). New York: Guilford Publications.

Oxford English Dictionary (1987). Oxford, UK: Oxford University Press.

Páez, D., Fernández, I. \& Martín Beristain, C. (2001). Catástrofes, traumas y conductas colectivas: procesos y efectos culturales. In C. San Juan (Ed.), Catástrofes y ayuda en emergencia: estrategias de evaluación, prevención y tratamiento (pp. 85-148). Barcelona: Icaria.

Puy, A. \& Romero, A. (1998). Claves para la intervención psicosocial en desastres. In A. M. Gonzáles (Ed.), Psicología comunitaria: fundamentos y aplicaciones (pp. 497-515). Madrid: Sintesis.

Quarantelli, E. L. (1985). What is disaster? The need for clarification in definition and conceptualization in research. In B. Sowder, Disasters and mental health selected 
contemporary perspectives (pp. 41-73). Washington, D. C.: Government Printing Office.

Quarantelli, E. L. (1998). Introduction: the basic question, its importance, and how is addresses in this volume. In E. L. Quarantelli, What is a disaster? Perspective on the question (pp. 01-7). London/New York: Routlege.

Reyes, G. (2006a). Foreword. In G. Reyes, \& G. A. Jacobs (Eds.), Handbook of international disaster psychology: fundamentals and overview (pp. 13-14). Westport, CT: Praeger.

Reyes, G. (2006b). Conclusions and recommendations for further progress. In G. Reyes, \& G. A. Jacobs (Eds.), Handbook of international disaster psychology: interventions with special needs populations (pp. 141-149). Westport, CT: Praeger.

Reyes, G. (2006c). Psychological first aid: principles of community-based psychosocial support. In G. Reyes, \& G. A. Jacobs, Handbook of international disaster psychology: practices and programs (pp. 01-12). Westport, CT/London: Praeger.

Staniford, A. K., Dollard, M. F. \& Guerin, B. (2009). Stress and help seeking for drought-stricken citrus growers in the Riverland of South Australia. Rural Health, 17, 147-154.

Ünal-Karagüven, M. H. (2009). Psychological impact of an economic crisis: a Conservation of Resources Approach. International Journal of Stress Management, 16(3), 177194.

Recebido em 06/08/2013 Aceito em 15/07/2014

Eveline Favero: professora adjunta de Psicologia na Universidade Estadual do Oeste do Paraná, Brasil.

Jorge Castellá Sarriera: professor do Programa de Pós-Graduação em Psicologia da Universidade Federal do Rio Grande do Sul, Brasil.

Melina Carvalho Trindade: psicóloga, especialista em Terapia de Família e de Casal e em Terapia CognitivoComportamental, membro do Domus - Centro de Terapia de Casal e de Família, Brasil. 